\title{
Asset Trading Mechanisms and Expansionary Policy*
}

\author{
DAVID K. LEVINE \\ Department of Economics, University of California, \\ Los Angeles, California 90024-1477
}

Received January 22, 1990; revised September 17, 1990

\begin{abstract}
Previous authors have argued that the otimal monetary policy is contractionary. If buyers value consumption substantially more than sellers, there is some randomness and informational constraints make asset trading useful, we show that there is an incentive compatible expansionary policy that dominates all incentive compatible contractionary policies. (01991 Academic Press, Inc.
\end{abstract}

\section{INTRODUCTION}

Friedman [5], Bewley [2, 3], and Townsend [21] argue that the optimal monetary policy is contractionary. We give a simple example showing that if buyers value consumption more than sellers by a sufficient margin and there is some randomness in the economy, then there is an expansionary policy that dominates all contractionary policies. Intuitively, if the lump sum taxes that are needed to finance a deflation cannot differentiate buyers and sellers, they will make it difficult for an unlucky buyer (who has little money) to consume. An expansion, on the other hand, will tend to insure unlucky buyers.

The goals of this paper are two: to establish the validity of the intuitive argument, and to explain why lump sum taxes should not differentiate between buyers and sellers. If they could, there would be no need for money: the government could carry out intertemporal and interstate trade directly through taxes. To formalize this idea we give a mechanism

* Part of this paper was written while at the Federal Reserve Bank of Minneapolis and the University of Minnesota. This paper benefited substantially from discussion with V. V. Chari, Drew Fudenberg, Patrick Kehoe, Robert Lucas, Joseph Ostroy, Edward Prescott, Robert Townsend, and above all Michael Woodford. I am also grateful to Roger Guesnerie, Frank Hahn, Timothy Kehoe, Andreu Mas-Colell, Thomas Sargent, Jose Scheinkman, Guido Tabellini, participants of the Theory Workshops at UCLA and Cal Tech, and the Money workshops at the University of Chicago, UC San Diego, and the University of Minnesota, and to two anonymous referees. NSF Grant SES 87-08616 and 89-07999 provided financial support. 
theoretic rationale for the Townsend/Bewley model, arguing that if buyers and sellers cannot be identified this both provides a rationale for a monetary mechanism and forces the conclusion that lump sum taxes cannot differentiate between buyers and sellers.

To keep the analysis simple, we examine an example in which two types of infinitely lived agents shift randomly back and forth between being buyers and sellers. With each agent's type private information, we consider mechanisms in which information is carried between periods by the private holding of divisible tradable assets. If a first best is achievable by such a mechanism, it is achievable with just one asset, which we interpret as money. The mechanism itself we interpret as a government policy. We can then focus on the nature of mechanisms that achieve the first best. A contractionary policy pays a positive rate of interest on money balances or contracts the money supply; an expansionary policy taxes money balances or expands the money supply. We show that if there is some randomness in the economy, no contractionary policy can ever achieve the first best. On the other hand, if agents are sufficiently patient and the desire to trade sufficiently strong, the first best can be achieved by a flat expansion: an expansion in which the tax on money balances and rate of expansion of the money supply are constant independent of time, state, and history. Related results have been shown by Scheinkman and Weiss [19], who show a one-time increase in the money stock can be welfare improving, and by Woodford [24], who shows the potentially beneficial redistributive effects of an expansion in a model of transactions costs.

By focusing on the case where there are two types and two states, the analysis is simplified because it is possible to implement the first best with a single asset. With more states and/or types the first best may be unobtainable by any mechanism, and mechanisms with several assets may dominate a mechanism with a single asset. To analyze such a world would require a more difficult second best analysis, and would also have to deal with some ambiguity about what would constitute an "expansionary" or "contractionary" policy with more than one asset. Nevertheless, the central economic theme of this paper that in the presence of incentive constraints, redistributional effects are ubiquitous remains valid. Moreover, inflation will transfer wealth from traders who hold large money balances to those that hold smaller balances. To the extent that this redistribution alleviates the insurance problem created by incentive constraints, it will tend to improve welfare.

Our results stand in contrast to Friedman's [5] theory of the optimal quantity of money as developed by Bewley $[2,3]$ and Townsend [21]. A good summary of this work can be found in Sargent [18]. A similar result in a different but related model can be found in Taub [20]. They suggest that in order to increase the real value of the money stock, a contraction 
is desirable. However, when the future is uncertain, private individuals will optimally plan to run out of money with positive probability, preventing them from buying when it is socially desirable that they do so. This can be prevented only by an expansion. Moreover, unlike the Bewley/Townsend model, where the first best requires that the marginal utility of buyers and sellers be equalized, here the first best forces sellers to zero consumption. This allows the marginal utility of buyers to be strictly higher than that of sellers. Because of this gap, the wedge between the marginal utility of buyers and sellers caused by a modest rate of inflation does not reduce trade. When the first best equalizes the marginal utility of buyers and sellers, Kehoe, Levine, and Woodford [10] show that there is a tradeoff between the beneficial redistributional effect of an expansion, and the reduction in trade due to the increased wedge between the marginal utility of buyers and sellers.

It is important to emphasize that we do not assume that a monetary expansion is the only source of government revenue: indeed, we permit mechanisms in which the entire stock of consumption is controlled by the government. Without private information, the first best can be achieved simply by redistributing all consumption to buyers: money and assets are not required. With private information, the only incentive compatible redistribution scheme requires some expansion. The key point is that the same feature of the model that makes money interesting forces an expansionary policy-it is not true that this redistribution could be achieved by a mix of deflation and some other policy.

\section{THE ENVIRONMENT}

There are two types of infinitely lived agent, denoted type 1 and type 2 . There are a continuum of agents, with each type constituting half the population. An individual agent's type is known only to himself.

There are two possible states of the world, denoted state 1 and state 2 . These states follow a Markov chain, with $\pi$ denoting the probability that the state changes from 1 to 2 or 2 to 1 , and $1-\pi$ denoting the probability that the state remains the same. The probability $\pi$ is referred to as the probability of reversal. The state of the world in period $t$ is denoted $\eta_{t}$. The initial state $\eta_{1}$ has equal probability of being either state 1 , or state 2 .

There is a single perishable consumption good $x$. One unit of this good is available per capita each period. If $x_{t}^{a}$ denotes the consumption of agent $a$ at time $t$, his preferences have the additively separable form

$$
U\left(x^{a}\right) \equiv E \sum_{t=1}^{\infty} \delta^{t-1} u^{a}\left(x_{t}^{a}, \eta_{t}\right)
$$


where $0<\delta<1$ is a subjective discount factor common to all types, and $E$ denotes the initial expectation before $\eta_{1}$ is known. The period utility function $u^{a}\left(x_{t}^{a}, \eta_{t}\right)$ is $\bar{u}\left(x_{t}^{a}\right)$ if the state $\eta_{t}$ is the same as agent $a$ 's type, and $\underline{u}\left(x_{t}^{a}\right)$ if not. We always assume:

(A.1) $\bar{u}\left(x_{t}^{a}\right), \underline{u}\left(x_{t}^{a}\right)$ are bounded, increasing, concave, and continuously differentiable.

Boundedness is a technical assumption ensuring "continuity at infinity" in the sense of Fudenberg and Levine [6] that the future does not matter too much; monotonicity and concavity are standard. Differentiability is convenient for notational simplicity. One special case of some importance is the case of a linear environment: for some $\varepsilon, \bar{\eta}, \eta>0, \bar{u}\left(x_{t}^{a}\right)=\bar{\eta} x_{t}^{a}$, and $\underline{u}\left(x_{t}^{a}\right)=\eta x_{t}^{a}$ for $0 \leqslant x_{t}^{a} \leqslant 2+\varepsilon$. Since when all agents of a given type are treated equally no agent can consume more than 2 , this means that the utility functions are linear in the relevant range. Notice that since they are bounded above, $\bar{u}$ and $\underline{u}$ cannot be linear globally.

We should emphasize the convention that the total mass of agents in the economy is $1: 1 / 2$ the population is of type 1 and $1 / 2$ is of type 2 . Consequently social feasibility means that $(1 / 2) x_{t}^{1}+(1 / 2) x_{t}^{2} \leqslant 1$.

As the economy shifts back and forth between states, the two types of agent shift back and forth between the period utility functions $\bar{u}$ and $\underline{u}$. When a type has utility $\bar{u}$, we refer to them as buyers, when the utility is $\underline{u}$, we refer to them as sellers. To justify this terminology, we assume

(A.2) $D \bar{u}(2)>D \underline{u}(0)$,

where $D \bar{u}, D \underline{u}$ represent the derivatives of $\bar{u}$ and $\underline{u}$, respectively. This says that when all of the single good is shared equally among buyers (each receiving two units, and each seller none) the marginal utility when a buyer is never-the-less greater than that when a seller.

Because both types are equally likely to begin life as a buyer, there is a unique ex ante efficient allocation mechanism in which all agents of a given type are treated equally. We refer to this as the best allocation, and from (A.2) it consists of dividing all of the single good equally among the buyers, each receiving two units, and each seller none. Notice that there are many efficient allocation mechanisms if we do not adapt the ex ante criterion that utility is evaluated where both types have an equal likelihood of beginning life as buyer or seller. In effect, having ex ante identical agents is equivalent to having transferable utility. The adoption of an ex ante criterion, while a strong assumption, is not an essential limitation of the analysis. In showing that the best allocation can be implemented, we have shown (obviously) the possibility of implementing an ex post first best allocation. On the other hand, as we point out below, the argument that the best allocation cannot 
be implemented by a contractionary policy easily operates to show that no ex post first best allocation can be implemented by a contraction either.

As we shall show in the next section, the best allocation is not always incentive compatible. However, using it as a benchmark substantially simplifies our analysis: it is easier to check whether or not the best allocation is incentive compatible than it is to calculate the constrained optimum.

\section{Asset Trading Mechanisms}

In this section we define mechanisms and asset trading mechanisms. We give necessary and sufficient conditions for the best allocation to be implementable. We show that trading constrains mechanism design by replacing nonmarginal decisions with marginal ones. Finally we show that in our environment only one asset is required. The nature of mechanisms that implement the best allocation is discussed below.

Our notion of implementability is that of open-loop Nash equilibrium in an anonymous decentralized game form. Let $\Sigma$ be an abstract space of actions. An open-loop strategy is a sequence of maps $\sigma_{t}\left(\tau, \eta_{1}, \ldots, \eta_{t}\right)$ mapping an agents type $\tau$ and the history of states to actions. A game form is a sequence of maps $f_{t}\left(\eta_{1}, \ldots, \eta_{t}, \sigma_{1}, \ldots, \sigma_{t}\right) \geqslant 0$ mapping the history of states and an agent's actions to nonnegative consumption levels. Hammond [8] refers to such a game form, in which an agent's consumption is independent of the play of other agents, as decentralized. The best allocation is implemented by the mechanism $f$ and strategy $\sigma$ if

$$
\begin{aligned}
f_{t}\left(\eta_{1}, \ldots, \eta_{t}, \sigma_{1}(\tau), \ldots, \sigma_{t}\left(\tau, \eta_{1}, \ldots, \eta_{t}\right)\right) & =2, & & \eta_{t}=\tau \\
& =0, & & \eta_{t} \neq \tau,
\end{aligned}
$$

so that buyers get two and sellers zero, and such that for all $\tilde{\sigma}$

$$
\begin{aligned}
& {[\bar{u}(2)+\underline{u}(0)] / 2(1-\delta)} \\
& \quad \geqslant E \sum_{t=1}^{\infty} \delta^{t-1} u^{a}\left(f_{t}\left(\eta_{1}, \ldots, \eta_{t}, \tilde{\sigma}_{1}(\tau), \ldots, \tilde{\sigma}_{t}\left(\tau, \eta_{1}, \ldots, \eta_{t}\right)\right), \eta_{t}\right)
\end{aligned}
$$

so that the utility from the best allocation cannot be improved upon by playing an alternative strategy $\tilde{\sigma}$. In other words, there must be an open-loop Nash equilibrium that yields the best allocation.

This definition of implementability is equivalent to both Nash and subgame perfect implementability in a general anonymous game. Anonymity means that no agent can respond to a deviation by a measure zero group 
of agents. Since the response to deviations by more than one agent is irrelevant to the determination of equilibrium, this implies the relevant space of strategies are open-loop. Moreover, because each agent is infinitesimal, an open-loop equilibrium in which no agent reacts to another's deviation is also a closed-loop or subgame perfect equilibrium: failing to respond to deviations is itself an equilibrium. Fudenberg and Levine [7] prove the converse: if a closed-loop equilibrium fails to be open-loop, then a switch from one equilibrium to another must be triggered by the deviation of a single agent, violating anonymity.

Given open-loop strategies and anonymity, decentralization of the game form merely simplifies notation. Given the equilibrium strategies of all agents, anonymity implies that any pair of agents playing the same strategy receives the same allocation. Determination of equilibrium depends only on what happens to an agent when he changes his individual strategy. The way in which allocations change when the entire distribution of strategies change is irrelevant and may be conveniently suppressed by a decentralized game form.

An alternative view of this type of mechanism is provided by Roberts [17]. He points out that we may strengthen the equilibrium concept to dominant strategies, provided that we require the mechanism yield socially feasible allocations only in equilibrium. In this context Hammond [8] shows that with a continuum of agents decentralization does not limit the allocations that can be achieved.

In general, a game form must keep track of the entire history of play of all agents. To maintain such records centrally is expensive. Townsend $[21,22]$ focuses on a spatial interpretation in which the physical movement of records between locations is costly, and Manuelli and Sargent [15] show that this interpretation is possible here. However, it is essential only that the storage of records through time is costly. Either interpretation leads us to focus on mechanisms in which record keeping is decentralized. An asset game form consists of a finite number $k$ of assets and a sequence of maps $F_{t}\left(\eta_{1}, \ldots, \eta_{t}, M_{t-1}^{a}, \sigma_{t}\right) \in \mathbb{R}_{+}^{k+1}$ mapping the history of states, and an agent's vector of revealed initial asset holdings $M_{t-1}^{a} \in \mathbb{R}_{+}^{k}$ and action $\sigma_{t}$ to pairs $\left(M_{t}^{a}, x_{t}\right)$, with $M_{t}^{a} \in \mathbb{R}_{+}^{k}$ final asset holdings and $x_{t} \in \mathbb{R}_{+}$consumption. For notational convenience, we let $M_{0}^{a}$ be the null symbol. In other words, at time 1 agents are issued consumption and $k$ different kinds of certificates. In subsequent periods, the mechanism depends on the history of an agent's actions only through the certificates he was given at the end of the previous period. Notice, however, that each period dated certificates are issued: agents do not have the option of increasing their supply of next period certificates by saving current certificates. Since certificates are physical entities, they must be issued and held in nonnegative quantities. We shall always assume 
(A.3) Free disposal. If $0 \leqslant y \leqslant F_{t}\left(\eta_{1}, \ldots, \eta_{t}, M_{t-1}^{a}, \sigma_{t}\right)$, then there exists $\sigma_{t}^{\prime}$ with $y=F_{t}\left(\eta_{1}, \ldots, \eta_{t}, M_{t-1}^{a}, \sigma_{t}^{\prime}\right)$.

In particular, agents cannot be prevented from discarding certificates; they must be held voluntarily.

Our first theorem shows that there is no loss in restricting attention to asset mechanisms. Define the constant

$$
\delta_{\pi} \equiv \frac{\delta \pi}{1-\delta(1-\pi)}
$$

This is the expected present value of a unit of utility received after the next reversal.

Proposition 3.1. The best allocation can be implemented by a mechanism if and only if it can be implemented by a single asset mechanism if and only if

$$
\frac{\bar{u}(2)-\bar{u}(0)}{\underline{u}(2)-\underline{u}(0)} \geqslant \delta_{\pi}^{-1} .
$$

Proof. If the best allocation is to be implemented, it is clear from anonymity that initial buyers and sellers must prefer the plan of receiving 2 when a buyer, 0 when a seller, to misrepresenting as the opposite type and getting 0 when a buyer and 2 when a seller. We may assume without loss of generality the initial state is $\eta_{1}=1$. A simple calculation shows that the expected value to an agent receiving utility $u_{1}$ in state 1 and $u_{2}$ in state 2 is

$$
\left\{\delta_{\pi} /\left(1-\delta_{\pi}^{2}\right)\right\}\left(u_{1}+\delta_{\pi} u_{2}\right)
$$

Consequently, it must be that $\bar{u}(2)+\delta_{\pi} \underline{u}(0) \geqslant \bar{u}(0)+\delta_{\pi} \underline{u}(2)$ for initial buyers and $\underline{u}(0)+\delta_{\pi} \bar{u}(2) \geqslant \underline{u}(2)+\delta_{\pi} \bar{u}(0)$ for initial sellers. Because $0<\delta_{\pi}<1$ and by (A.2), the former inequality is true if the latter is; that is, initial buyers do not wish to misrepresent if initial sellers do not. Rewriting the constraint for initial sellers yields (3.2).

It remains to construct a single asset mechanism that implements the best allocation when (3.2) holds. Consider the strategy space in which each agent may announce either that he is a buyer or a seller. Suppose without loss of generality $\eta_{1}=1$. Whenever $\eta_{t}=1$ agents who announce they are buyers get 2 units of consumption, sellers get 2 period $t$ certificates. Whenever $\eta_{t}=2$ agents with 2 period $t-1$ certificates get 2 units of consumption and 2 period $t$ certificates; agents who do not have 2 period $t-1$ certificates get nothing.

Each agent faces four possible states: he holds either 2 certificates or none; and $\eta_{t}$ is either 1 or 2 . The only choice is what to do when in state 
1: take 2 units of consumption or 2 certificates. In state 2 , noncertificate holders can get nothing, and free disposability implies certificate holders should take the full amount offered. With finitely many states and choices, and discounting, dynamic programming shows an optimum exists and is stationary. The only stationary plans are to get 2 in state 1,0 in state 2 , or vice versa. But we showed above that if (3.2) holds, both types prefer to consume when they are buyers.

In general, an asset mechanism can prevent agents from trading assets. To the extent that the mechanism is nonlinear, agents will have an incentive trade with each other. Following Townsend [22] and Hammond [8], we assume that preventing trade is prohibitively expensive. In addition to assuming that assets are infinitely divisible, two assumptions are involved:

(A.4) Ex post trade. For each $t$ and $\eta_{1}, \ldots, \eta_{t}$ there exists a $p \in \mathbb{R}_{+}^{k+1}$ such that if $z \in \mathbb{R}^{k+1}, p \cdot z=0$ and $F_{t}\left(\eta_{1}, \ldots, \eta_{t}, M_{t-1}^{a}, \sigma_{t}\right)+z \geqslant 0$ (and in particular represents a feasible plan) then there exists $\sigma_{t}^{\prime}$ such that

$$
F_{t}\left(\eta_{1}, \ldots, \eta_{t}, M_{t-1}^{a}, \sigma_{t}^{\prime}\right) \geqslant F_{t}\left(\eta_{1}, \ldots, \eta_{t}, M_{t-1}^{a}, \sigma_{t}\right)+z .
$$

In other words, there is a price $p$ such that agents can trade assets and consumption. Consequently, the mechanism must promise to deliver at least as much as agents could obtain through trade. A careful discussion of the way in which side-markets lead to this conclusion can be found in Hammond [8].

The second assumption concerning tradability is

(A.5) Ex ante trade. For all $t, \eta_{1}, \ldots, \eta_{t}, \sigma_{t}, \sigma_{t}^{\prime}$ and all $M_{t}^{a}, M_{t}^{a^{\prime}}, \lambda \in \mathbb{R}$ such that $\lambda M_{t}^{a}+(1-\lambda) M_{t}^{a^{\prime}} \geqslant 0$, there exists a $\sigma_{t}^{\prime \prime}$ such that

$$
\begin{gathered}
\lambda F_{t}\left(\eta_{1}, \ldots, \eta_{t}, M_{t}^{a}, \sigma_{t}\right)+(1-\lambda) F_{t}\left(\eta_{1}, \ldots, \eta_{t}, M_{t}^{a^{\prime}}, \sigma_{t}^{\prime}\right) \\
\quad=F_{t}\left(\eta_{1}, \ldots, \eta_{t}, \lambda M_{t}^{a}+(1-\lambda) M_{t}^{a^{\prime}}, \sigma_{t}^{\prime \prime}\right) .
\end{gathered}
$$

In other words, the dependence of $F_{t}$ on $M_{t}^{a}$ is linear affine. Townsend [22] discusses this type of assumption at length. It follows from allowing agents to trade initial assets before turning them in for final assets and consumption. If the $F_{t}$ schedule is not convex, agents with different asset holdings will pool them; if it is not concave, agents with the same holdings will redistribute them unequally. Consequently $F_{t}$ must be both concave and convex: that is, linear affine. The difference between this assumption and assumption (A.4) is that the trade in (A.4) involves simultaneous exchange of equal value; the trade in (A.5) involves trading assets before the mechanism operates for goods and assets later. One way to discourage this type of trade is by refusing to honor such contracts. Nevertheless, as a matter of practice, people will engage in some trade to avoid nonlinear 
tax schemes or price discrimination schemes (which are what (A.5) rules out), and some costs will be incurred in the process. Assumption (A.5) is an extreme assumption, but probably no worse than the other extreme assumption-that no ex ante trade is possible.

Finally we assume

(A.6) Closedness. If $y^{n}=F_{t}\left(\eta_{1}, \ldots, \eta_{t}, M_{t}^{a n}, \sigma_{t}^{n}\right)$ and $y^{n} \rightarrow y$, $M_{t}^{a n} \rightarrow M_{t}^{a}$, then there is a $\sigma_{t} \in \Sigma$ such that

$$
y=F_{t}\left(\eta_{1}, \ldots, \eta_{t}, M_{t}^{a}, \sigma_{t}\right) .
$$

This says that the set of achievable allocations is closed. Since any allocation that can be supported can still be supported when we take the closure of the set of achievable allocations, this is a technical assumption. It ensures that individual optima exist.

When (A.3) to (A.6) are satisfied, we refer to the mechanism as an asset trading mechanism. The force of this restriction is given by Proposition 3.2.

Proposition 3.2. The following three conditions are equivalent:

(i) the best allocation can be implemented by an asset trading mechanism

(ii) the best allocation can be implemented by a single asset trading mechanism

(iii) $D \bar{u}(2) / D \underline{u}(0) \geqslant \delta_{\pi}^{-1}$.

Proof. Follows from Lemmas 3.3 and 4.3 below.

Condition (iii) is similar to condition (3.2) in Proposition 3.1, except that average utility is replaced with marginal utility. When utility is linear, Proposition 3.2 implies the best allocation can be implemented by an asset trading mechanism if and only if it can be implemented by some mechanism. Roughly, trading prevents the mechanism from exploiting the concavity of utility by forcing nonmarginal decisions.

From Proposition 3.1, we know that (iii) is necessary for implementability of the best allocation in the linear case. In a nonlinear environment, we may define a corresponding linear environment in which the utility functions are linear for $0 \leqslant x_{t}^{a} \leqslant 2+\varepsilon$, and the slopes are $\bar{\eta}=D \bar{u}(2)$ and $\underline{\eta}=D \underline{u}(0)$. The necessity of (iii) then follows from.

LEMMA 3.3. If an asset trading mechanism implements the best allocation it does so also in the corresponding linear environment.

Proof. Let $F, \sigma$ be the asset trading game form and strategy implementing the best allocation, and suppose it does not implement the best allocation 
in the corresponding linear environment. Then there must be a strategy $\tilde{\sigma}$ that does better than $\sigma$. Let $\tilde{x}^{a}$ denote the consumption plan achieved by playing $\tilde{\sigma}$ using the game form $F$, and let $\hat{x}^{a}$ denote the best allocation. Recall that $U\left(x^{a}\right)$ denotes the expected present value in the original (nonlinear environment). Let $U^{L}\left(x^{a}\right)$ be the expected present value in the corresponding linear environment. By hypothesis $U^{L}\left(\tilde{x}^{a}\right)>U^{L}\left(\hat{x}^{a}\right)$. We will construct an allocation $x_{\dot{\lambda}}^{a}$, achievable by means of strategy $\sigma_{\dot{\lambda}}$, such that $U\left(x_{\hat{\lambda}}^{a}\right)>U\left(\hat{x}^{a}\right)$, contradicting the fact that $F$ and $\sigma$ implement $\hat{x}^{a}$.

First observe that because of discounting, the plan $x_{T}^{a}$, equal to $\tilde{x}^{a}$ if $t \leqslant T$ and equal to 0 if $t>T$, satisfies $U^{L}\left(x_{T}^{a}\right)>U^{L}\left(\hat{x}^{a}\right)$ for some sufficiently large $T$. Fix such a $T$. By free disposability there exists a strategy $\sigma_{T}$ such that $F$ yields $x_{T}^{a}$ when $\sigma_{T}$ is played.

Next, suppose $0 \leqslant \lambda \leqslant 1$. Since $\hat{x}^{a}$ and $x_{T}^{a}$ can be achieved by strategies $\hat{\sigma}$ and $\sigma_{T}$, it follows from (A.3) and (A.4) that $x_{\lambda}^{a} \equiv(1-\lambda) \hat{x}^{a}+\lambda x_{T}^{a}$ can be obtained by playing some strategy $\sigma_{\%}$. Since the plans $\hat{x}^{a}$ and $x_{T}^{a}$ are uniformly bounded over time and state, Taylor's theorem applied to $u\left(x_{t}^{a}, \eta_{t}\right)$ implies

$$
U\left(x_{\lambda}^{a}\right)-U\left(\hat{x}^{a}\right)=\lambda U^{L}\left(x_{T}^{a}\right)-\lambda U^{L}\left(\hat{x}^{a}\right)+o(\lambda),
$$

where $o(\lambda) / \lambda \rightarrow 0$ as $\lambda \rightarrow 0$. But then $U^{L}\left(\hat{x}^{a}\right)-U^{L}\left(x_{T}^{a}\right)<0$ implies $U\left(\hat{x}^{a}\right)-$ $U\left(x_{\lambda}^{a}\right)<0$ for all $\lambda$ small enogh, the desired contradiction.

The sufficiency of a single asset trading mechanism is proven below in Section 4 as

LEMMA 3.4. If $D \bar{u}(2) / D \underline{u}(0) \geqslant \delta_{\pi}^{-1}$, then there is a single asset trading mechanism that implements the best allocation.

\section{Single Asset Trading Mechanisms}

One aspect of both Propositions 3.1 and 3.2 is that they show that no more than one asset is needed. From consideration of market completeness, such as those of Arrow [1] or Duffie and Huang [4], we see that no more assets are needed than states (two). On the other hand, since assets may be tailored to individuals, we would also expect that no more assets are needed than people (two). However, we have shown that we can eliminate one asset: roughly, we can tailor assets to all except one type, and give that type whatever is left over.

Assets are expensive. They are costly to issue, and verification is required to avoid forgery. Moreover, the markets in which they are traded are not costless to operate. Because of these transactions costs, it is desirable for asset trading mechanisms to use as few assets as possible. For this reason, 
we now focus on the case of a single asset, which we refer to as money. Throughout the rest of the paper, then, $\boldsymbol{M}_{t}^{a}$ will be a scalar denoting agent $a$ 's holdings of money at $t$. Our ultimate goal is to partially characterize single asset trading mechanisms that can support the best allocation. We do so in terms of whether or not they are contractionary or expansionarythat is, whether the per capita money supply increases or decreases. Our goal is to show that no contractionary mechanism can support the best allocation, but that an expansionary mechanisms sometimes can.

We first characterize single asset trading mechanisms that can implement the best allocation.

Proposition 4.1. The best allocation can be implemented by a single asset trading mechanism if and only if there exist nonnegative prices $p_{M}\left(\eta_{1}, \ldots, \eta_{t}\right)$, $p_{x}\left(\eta_{1}, \ldots, \eta_{t}\right), q\left(\eta_{1}, \ldots, \eta_{t}\right)$ and lump sum subsidies $L\left(\eta_{1}, \ldots, \eta_{t}\right) \geqslant 0$ such that agent $a$, when faced with initial asset holdings of $M_{0}^{a} \equiv 0$ and the sequence of budget constraints,

$$
p_{M}\left(\eta_{1}, \ldots, \eta_{t}\right) M_{t}^{a}+p_{x}\left(\eta_{1}, \ldots, \eta_{t}\right) x_{t}^{a} \leqslant q\left(\eta_{1}, \ldots, \eta_{t}\right) M_{t-1}^{a}+L\left(\eta_{1}, \ldots, \eta_{t}\right),
$$

finds it optimal to consume 2 units when a buyer and 0 when a seller.

\section{Proof. See the Appendix.}

Note that $L\left(\eta_{1}, \ldots, \eta_{t}\right)$ is independent of type. In addition, these budget constraints are exactly those in a Bewley/Townsend style monetary economy in which the government is restricted to controlling the growth of the money supply using equal lump sum taxes. Consequently, when the best allocation is implementable by a single asset trading mechanism, it may be interpreted as a monetary equilibrium in a Bewley/Townsend economy.

Incentive compatibility forces the price of goods to be strictly positive, so we take goods to be numeraire. We can then rewrite (4.1) as

$$
\rho^{t} m_{t}^{a}+x_{t}^{a} \leqslant \rho_{t}\left(R_{t} \phi_{t}^{-1}\right) m_{t-1}^{a}+l_{t},
$$

where if $\bar{M}_{t}$ denotes the per capita money stock, $m_{t}^{a} \equiv M_{t}^{a} / \bar{M}_{t}$ is agent $a$ 's share; where $R_{t} \equiv q\left(\eta_{1}, \ldots, \eta_{t}\right) / p_{M}\left(\eta_{1}, \ldots, \eta_{t}\right)$ is the gross nominal interest paid on initial money holdings; $\rho_{t} \equiv\left[p_{M}\left(\eta_{1}, \ldots, \eta_{t}\right) / p_{x}\left(\eta_{1}, \ldots, \eta_{t}\right)\right] \bar{M}_{t+1}$ is the real value of the end of period money stock; $\phi_{t} \equiv \bar{M}_{t+1} / \bar{M}_{t}$ is the gross growth rate of the money stock; and $l_{t} \equiv L\left(\eta_{1}, \ldots, \eta_{t}\right) / p_{x}\left(\eta_{1}, \ldots, \eta_{t}\right)$ is the real value of the lump sum subsidy (again, independent of type). Note that with the money stock normalized to one $\rho_{t}$ is also the goods price of money. As with goods, since $m_{t}^{a}$ is a per capita measure, and the population is 1 , social feasibility means $(1 / 2) m_{t}^{1}+(1 / 2) m_{t}^{2} \leqslant 1$. 
To prove that asset trading mechanism of various types exist, we must give examples of price systems such that the hypotheses of Proposition 4.1 are satisfied. To check for optimality, it is convenient to use first order conditions. To do so, we must introduce notation to distinguish between the two states that can occur next period. If $y_{t}$ is a time $t$ realization, let $y_{t+1}$ be the realization at time $t+1$ if the state is the same at $t+1$ as at $t$, and let $\tilde{y}_{t+1}$ denote the realization if a reversal occurs.

LEMMA 4.2. Given budget constraints of the form (4.2) it is sufficient for the best allocation to be optimal that there exist a budget feasible contingent plan for money holding $m_{t}^{a}$, and nonnegative contingent marginal utilities of income $\mu_{t}^{a}$ and a constant $B$ so that the first order conditions

$$
\begin{gathered}
\mu_{t}^{a}=D \bar{u}(2) \quad \text { if } a \text { is a buyer at } t \\
\geqslant D \underline{u}(0) \quad \text { if } a \text { is seller at } t \\
\mu_{t}^{a} \rho_{t} \geqslant \delta\left[(1-\pi) \rho_{t+1}\left(R_{t+1} \phi_{t+1}^{-1}\right) \mu_{t+1}^{a}+\pi \tilde{\rho}_{t+1}\left(\tilde{R}_{t+1} \tilde{\phi}_{t+1}^{-1}\right) \tilde{\mu}_{t+1}\right] \\
\left(=\text { if } m_{t}^{a}>0\right)
\end{gathered}
$$

and the transversality condition,

$$
\mu_{t}^{a} \rho_{\imath} \leqslant B
$$

are satisfied.

We will not prove this lemma here: proofs may be found in Levine [14] or Bewley [2], and are a straightforward extension of the results of Weitzman [23] to the stochastic case. We merely observe that if $\pi\left(\eta_{1}, \ldots, \eta_{t}\right)$ is the probability of the history $\eta_{1}, \ldots, \eta_{t}$, then (4.3) are the first order conditions that follow from forming a Lagrangean by associating the multipliers $\delta^{\prime} \pi\left(\eta_{1}, \ldots, \eta_{t}\right) \mu_{t}^{a}$ with the budget constraint after the history $\eta_{1}, \ldots, \eta_{t}$. The transversality condition (4.4) requires that the marginal utility of a share of the money stock must be bounded. We can now complete the proof of Proposition 3.2 as

Lemma 4.3. If $D \bar{u}(2) / D \underline{u}(0) \geqslant \delta_{\pi}^{-1}$, then there is a single asset trading mechanism that implements the best allocation.

Proof. Suppose, without loss of generality, that $\eta_{t}=1$. At the end of every period assign type 2's the entire money stock, so that if $a$ is a type 2 at $t, m_{t}^{a}=2$. In periods in which type 2's are buyers, $\rho_{t}=(1-\pi) \delta_{\pi} / \pi$, $R_{t} \phi_{t}^{-1}=1 / \delta(1-\pi)$, and $l_{t}=0$. In periods (including the initial period) in which type 2's are sellers, $\rho_{t}=1, R_{t} \phi_{t}^{-1}=0$, and $l_{t}=2$. A check shows hat (4.2) is satisfied. It remains to give marginal utilities of expenditure for 
sellers. When type 2's are sellers, set $\mu_{t}^{a}=\delta_{\pi} D \bar{u}(2)$. When type 1's are sellers, set $\mu_{t}^{a}=D \underline{u}(0)$. The transversality condition (4.4) is obviously satisfied. A calculation shows that if $D \bar{u}(2) / D \underline{u}(0) \geqslant \delta_{\pi}^{-1},(4.3)$ is as well. Notice, incidentally, that this is essentially the same mechanism used in the proof of Proposition 3.1.

\section{Expansionary and Contractionary Policy}

A given mechanism must be enforced; it is natural to think of the enforcement agent as the government. The mechanism can be enforced either through policy-the rate at which the money supply grows; or through enforcement of private contracts-the enforcement of the payment of interest. If no interest is paid on money (so $R_{t}=1$ ) a policy is expansionary if $\phi_{t}>1$, so that the money supply grows, or contractionary if $\phi_{t} \leqslant 1$. In light of the fact that (4.2) implies that agents care only about $R_{t} \phi_{t}^{-1}$, this motivates the following distinction between mechanisms: A mechanism is expansionary if $\phi_{t} R_{t}^{-1}>1$ for all times and histories; a mechanism is contractionary if $\phi_{t} R_{t}^{-1} \leqslant 1$ for all times and histories.

Our goal is to characterize mechanisms ability to achieve the best allocation. In the case of contractionary mechanisms:

Proposition 5.1. If $0<\pi<1$, then no contractionary mechanism can implement the best allocation.

Proof. First we show $\rho_{t} \leqslant 3 D \bar{u}(2) /(1-\delta) D \underline{u}(0) \equiv C$ almost surely. Let $\hat{x}^{a}$ denote the best allocation. Suppose $\rho_{t}>C$ for some history $h_{t}=\left(\eta_{1}, \ldots, \eta_{t}\right)$. One type of agent holds at least one unit of money per capita, and can afford the consumption plan $\tilde{x}_{t}^{a}$ equal to $\hat{x}^{a}$ before $t$ or if $h_{t}$ does not occur, and equal to $C$ in period $t$ and zero forever afterwards if $h_{t}$ does occur. Consider the linear environment defined by $\bar{\eta} \equiv D \bar{u}(2), \underline{\eta}=D \underline{u}(0)$. Contingent on $h_{t}, \hat{x}^{a}$ gives a present value of no more than $2 \bar{\eta} /\left(1^{-}-\delta\right)$ units of utility at $t$; while $\tilde{x}^{a}$ gives $3 \bar{\eta} /(1-\delta)$. Since $0<\pi<1, h_{t}$ has positive probability, and $U^{L}\left(\tilde{x}^{a}\right)>U^{L}\left(\hat{x}^{a}\right)$. This contradicts Lemma 3.3, showing $\rho_{t} \leqslant C$.

Next observe that incentive compatibility implies (4.2) must hold with exact equality. Aggregating over agents this shows that $\rho_{t}+1=$ $\rho_{t} R_{t} \phi_{t}^{-1}+l_{t}$. Solving for $l_{t}$ and substituting into the buyer's budget constraint (4.2) yields

$$
\left(m_{t}^{a}-1\right)=R_{t} \phi_{t}^{-1}\left(m_{t-1}^{a}-1\right)-1 / \rho_{t} .
$$


By hypothesis in a contraction $\phi_{t} R_{t}^{-1} \leqslant 1$, so $R_{t} \phi_{t}^{-1} \geqslant 1$, and we just showed $-1 / \rho_{t} \leqslant-1 / C$. It follows that if $m_{t-1}^{a} \leqslant 1$ then

$$
\left(m_{t}^{a}-1\right) \leqslant\left(m_{t-1}^{a}-1\right)-(1 / C),
$$

and in particular $m_{t}^{a}<1$. Since $m_{t}^{a} \geqslant 0$, we see that it is impossible for an agent who has no more than the average money stock at the beginning of a period to be a buyer more than $C$ consecutive periods. Unfortunately, since $0<\pi<1$, this event occurs with positive probability. This contradiction establishes that a contractionary policy is inconsistent with the best allocation.

The key to this argument is that agents self-insure by holding money, as in Leland [12]. Consequently, if they have bad luck too many times in a row, and do not get help from the government they will no longer be able to buy. This idea can also be found in Leijonhufvud [11], where it is suggested that the economy will behave "classically" as long as there is enough liquidity in the system to act as a buffer. It should be noted that this result does not depend on the rather strong ex ante welfare criterion we have adopted. Any ex post efficient allocation must require one of the two traders to purchase a minimum increment to consumption when a buyer. Since it can be shown that $\rho_{t}$ must still be bounded above, it follows that a sufficiently long string of bad luck will still cause this buyer to run out of money.

A general method of computing equilibria of this type may be found in Kehoe and Levine [9]. Existence issues are discussed in Levine [13]. Equilibria of this type in a similar model have also been computed by Scheinkman and Weiss [19], who point out a one time unanticipated expansion can help when money balances are badly distributed. In this model, we can prove a much stronger result.

In case $\phi_{t} R_{t}^{-1}$ is independent of time and history we refer to a flat expansion or contraction. Such mechanisms are simpler and less costly to operate. In particular a flat expansion can be implemented by government helicopters dropping a fixed percent of the current money supply each period, and letting private markets take their course. We have the following strong converse to Propositions 5.1.

Proposition 5.2. Suppose

$$
D \bar{u}(2) / D \underline{u}(0) \geqslant 2 / \delta \pi+2 \delta_{\pi}^{-1} .
$$

Then the best allocation can be implemented by a flat expansion (of $\phi_{t} R_{t}^{-1}=3$ ). 
Proof. Let $R_{t} \phi_{t}^{-1}=1 / 3$. In the initial period the goods price of money $\rho_{1}=1$. Adding (4.2) across types shows that $l_{1}=1$. At the end of every period assign sellers the entire money stock, so that if $a$ is a seller at $t$, $m_{t}^{a}=2$ (each seller holds twice the average money stock). If at the beginning of the period buyers hold all the money $\rho_{t}=\bar{\rho} \equiv 3 / 4$; if at the beginning of the period sellers hold all the money $\rho_{t}=\underline{\rho} \equiv 3 / 2$. In either case aggregating (4.2) shows

$$
l_{t}=1+(2 / 3) \rho_{t} .
$$

By Lemma 4.4, it suffices to give marginal utilities of expenditure $\mu_{t}^{a}$ satisfying (4.3) and (4.4). For buyers (4.3) gives $\mu_{t}^{a}$. Set $\bar{\mu} \equiv\left[2 / \delta \pi+\delta_{\pi}^{-1}\right]^{-1} D \bar{u}(2)$. For sellers in the initial period, take $\mu_{1}^{a}=(3 / 4) \bar{\mu}$. In periods in which buyers initially hold all the money, take sellers marginal utility of expenditure to be $\mu_{t}^{a}=\bar{\mu}$, while if sellers initially hold all the money, take $\mu_{t}^{a}=(1 / 2) \bar{\mu}$. Since $\mu_{t}^{a}$ takes on only the values $D \bar{u}(2),(3 / 4) \bar{\mu}, \bar{\mu}$, and $(1 / 2) \bar{\mu}$, it clearly satisfies the transversality condition (4.4). A direct calculation shows that (5.1) implies the first order conditions (4.3).

Observe, incidently, that the bound in Proposition 4.3 is stronger than that in Proposition 3.2: We do not attempt to show that the best allocation can be implemented by a flat expansion whenever it can be implemented by some asset trading mechanism, nor do we attempt to find the best possible bound.

\section{APPENDIX}

Proposition 4.1. The best allocation can be implemented by a single asset trading mechanism if and only if there exist nonnegative prices $p_{M}\left(\eta_{1}, \ldots, \eta_{t}\right)$, $p_{x}\left(\eta_{1}, \ldots, \eta_{t}\right), q\left(\eta_{1}, \ldots, \eta_{t}\right)$ and lump sum subsidies $L\left(\eta_{1}, \ldots, \eta_{t}\right) \geqslant 0$ such that agent $a$, when faced with initial asset holdings of $M_{0}^{a} \equiv 0$ and the sequence of budget constraints,

$$
\begin{aligned}
& p_{M}\left(\eta_{1}, \ldots, \eta_{t}\right) M_{t}^{a}+p_{x}\left(\eta_{1}, \ldots, \eta_{t}\right) x_{t}^{a} \\
& \quad \leqslant q\left(\eta_{1}, \ldots, \eta_{t}\right) M_{t-1}^{a}+L\left(\eta_{1}, \ldots, \eta_{t}\right),
\end{aligned}
$$

finds it optimal to consume 2 units when a buyer and 0 when a seller.

Proof. Supposing that the best allocation is in fact optimal for each agent given $M_{0}^{a}=0$ and the sequence of budget constraints (4.1), we can design a game form in which each agents strategy $\sigma$ is to announce a desired ratio of final money to consumption (possibly infinity), and in which $F_{t}$ assigns the unique pair $\left(M_{t}^{a}, x_{t}^{a}\right)$ consistent with this ratio that 
satisfies (4.1) with exact equality. Together with the strategy of announcing truthfully, this mechanism clearly implements the best allocation.

Conversely, for a mechanism to implement the best allocation, it is clear that the plan of consuming 2 when a buyer and 0 when a seller must be optimal in the set of all consumption plans that can be generated by playing different strategies. From ex post tradability, (A.3), the set of final money holdings $M_{t}^{a}$ and consumption $x_{t}^{a}$ that are feasible for an agent $a$ with initial money holdings $M_{t-1}^{a}$ have the form

$$
\begin{aligned}
& p_{M}\left(\eta_{1}, \ldots, \eta_{t}\right) M_{t}^{a}+p_{x}\left(\eta_{1}, \ldots, \eta\right) x_{t}^{a} \\
& \quad \leqslant \max _{\sigma_{t} \in \Sigma} p\left(\eta_{1}, \ldots, \eta_{t}\right) F_{t}\left(\eta_{1}, \ldots, \eta_{t}, M_{t-1}^{a}, \sigma_{t}\right),
\end{aligned}
$$

where $p=\left(p_{M}, p_{x}\right)$ is the nonnegative price vector whose existence is asserted in (A.3) and the existence of a maximum is assured by (A.5). Moreover, from $e x$ ante tradability, (A.4), the right hand side of (1) is a linear affine function of $M_{t-1}^{a}$, and we may write

$$
\begin{array}{r}
\max _{\sigma_{t} \in \Sigma} p\left(\eta_{1}, \ldots, \eta_{1}\right) F_{t}\left(\eta_{1}, \ldots, \eta_{t}, M_{t-1}^{a}, \sigma_{t}\right) \\
=q\left(\eta_{1}, \ldots, \eta_{t}\right) M_{t-1}^{a}+L\left(\eta_{1}, \ldots, \eta_{t}\right) .
\end{array}
$$

Moreover, by free disposability of assets, $q$ is nonnegative while the fact that $F_{t}\left(\eta_{1}, \ldots, \eta_{t}, 0, \sigma_{t}\right) \geqslant 0$ implies that $L\left(\eta_{1}, \ldots, \eta_{t}\right) \geqslant 0$. Combining (1) and (2) yields $(4.1)$.

\section{REFERENCES}

1. K. ARrow, "Essays in the Theory of Risk Bearing," North-Holland, Amsterdam, 1974.

2. T. BEWLEY, The optimum quantity of money, in "Models of Monetary Economics" (J. Karaken and N. Wallace, Eds.), Federal Reserve Bank of Minneapolis, 1980.

3. T. BEWLEY, A difficulty with the optimum quantity of money, Econometrica 51 (1983), $1485-1504$.

4. D. Duffie and M. Huang, Stochastic production exchange economies, Stanford, 1986.

5. M. Friedman, "The Optimum Quantity of Money and Other Essays," Aldine, Chicago, 1969.

6. D. Fudenderg AND D. Levine, Subgame perfect equilibrium of finite- and infinite-horizon games, J. Econ. Theory 31 (1983), 251-268.

7. D. FUdENBERG AND D. LeVINE, Open and closed-loop equilibria in games with many players, J. Econ. Theory 44 (1988), 1-18.

8. P. Hammond, Straightforward incentive compatibility in large economies, Rev. Econ. Stud. 46 (1979), 263-292.

9. T. KeHOE AND D. LeVINE, Approximate Markov equilibria of stationary rational expectations models, UCLA, 1985. 
10. T. KeHOE, D. LeVINE, AND M. WoOdford, The optimum quantity of money revisited, in "Economic Analysis of Markets and Games" (Douglas Gale and Oliver Hart, Eds.), in press.

11. A. Lejonhufvud, Effective demand failures, Swedish J. Econ. 75 (1973), 27-48.

12. H. LELAND, Savings and uncertainty: The precautionary demand for savings, Quart. J. Econ. 82 (1968), 465-473.

13. D. LeVINE, Efficiency and the value of money, Rev. Econ. Stud. 56 (1989), 77-88.

14. D. LEVINE, Liquidity with random market closure, UCLA, 1986.

15. R. Manuelli and T. Sargent, "Longer Trading Periods in the Townsend Turnpike Model," Hoover Institute, Stanford, 1988.

16. T. RADNER, Existence of equilibrium of plans, prices, and price expectations in a sequence of markets, Econometrica 40 (1972).

17. K. RoBerTs, Theoretical limits to redistribution, Rev. Econ. Stud. 51 (1984), 177-195.

18. T. Sargent, "Dynamic Macroeconomic Theory," Harvard Univ. Press, Cambridge, 1987.

19. J. SCheinKman aND L. Weiss, Borrowing constraints and aggregate economic activity, Econometrica 54 (1986), 23-54.

20. B. TAUB, Efficiency in a pure currency economy with inflation, Econ. Inquiry 26 (1988), 567-583.

21. R. TownSEND, Models of money with spatially separated agents, in "Models of Monetary Economics" (J. Karaken and N. Wallace, Eds.), Federal Bank of Minneapolis, 1980.

22. R. TOWNSEND, "Optimal Activist Currency Rules," University of Chicago, 1987.

23. M. Weitzman, Quantity theory for infinite horizon convex models, Manage. Sci. 19 (1973).

24. M. WOODFORD, Transaction costs, liquidity and optimal inflation, in "Essays in Intertemporal Economics," Ph.D. dissertation, MIT, 1983. 